\title{
Presencia de mercurio en la especie Pimelodus grosskopfii “Capaz” en el departamento del Huila
}

\section{The presence of mercury in the long-whiskered catfish (Pimelodus grosskopfii) species in the department of Huila}

\author{
Ángela Goretty García Gómez ${ }^{[1]}$, Andrea Ximena Parejo Pérez ${ }^{[2]}$, Lina Margarita Vela Bonilla ${ }^{[3]}$ \\ y Lilia Vanesa Coronado Cuenca ${ }^{[4]}$
}

\begin{abstract}
Resumen
En el presente trabajo se realizaron mediciones para determinar la presencia de Mercurio orgánico (metilmercurio) en la especie Pimelodus grosskopfii “Capaz” en el Departamento del Huila; para ello se determinó el contenido de mercurio total de 35 muestras de peces los cuales fueron capturados entre finales de noviembre y principios de diciembre del año 2012 en los municipios de Hobo, Gigante, vereda de Puerto Seco, Yaguará y Neiva, presentando niveles de mercurio todos los especímenes capturados; adicional a esto se determinó el análisis de riesgo al cual puede estar expuesta la población sensible como son los pescadores, debido al consumo de esta especie, por medio del índice de riesgo Hazard quotient HQ reportando valores inferiores a uno.
\end{abstract}

Palabras clave: Análisis de riesgo, Pimelodus grosskopfii, Índice de riesgo, metil mercurio.

\begin{abstract}
This study measured the presence of organic mercury (methylmercury) in the Long-whiskered catfish (Pimelodus grosskopfii) species in the Huila department. To do this, we determined the total mercury content of 35 fish samples caught between the end of November and beginning of December 2012 in the municipalities of Hobo, Gigante, Yaguará, Neiva and the Puerto Seco district. All the samples presented levels of mercury. We also determined the fishing population's risk of mercury exposure due to the consumption of this species. To do this, we used the Hazard quotient HQ index, finding values inferior to one.
\end{abstract}

Key words: Risk analysis, Pimelodus grosskopfii, risk index, methylmercury.

1] MSc. en Ingeniería Civil con énfasis en Gestión Ambiental, Ingeniera química., Calle 8 No. 32-49. angela.garcia@ corhuila.edu.co

[2] Ingeniera Ambiental; Calle 8 No. 32-49; andreaximena1391@gmail.com

[3] Ingeniera Ambiental; Calle 8 No. 32-49; lina_m78@hotmail.com

[4] Ingeniera Ambiental; Calle 8 No. 32-49; lilivanecocu@gmail.com

Recibido: 15 diciembre 2014 • Aceptado: 20 abril 2015 


\section{Introducción}

El mercurio es un metal pesado que se puede acumular en los sedimentos en la base de los cuerpos de agua, en este punto los microorganismos como bacterias que viven allí pueden convertirlo a la forma orgánica; conocida como metilmercurio para luego ser acumulado por los peces y así llegar al hombre (Barceló 2000). Aunque las diferentes formas del mercurio, pueden afectar a las personas en diversas circunstancias; es el consumo de pescado contaminado con metilmercurio una de las principales vías de exposición (OMS 2012); el cual permite que aun en niveles bajos de exposición a esta sustancia, las personas o poblaciones pueden desarrollar alteraciones en las funciones del sistema nervioso, con consecuencias neurofisiológicas graves particularmente en la etapa de gestación y en los niños pequeños (Lebel, Roulet, Mergler, Lucotte, \& Larribe, 1996); (Xue J, 2011).

De acuerdo con datos del Departamento Norteamericano de Drogas y Alimentos (FDA) aproximadamente 2.700 a 6.000 toneladas de mercurio son arrojadas anualmente a la atmósfera por gasificación de la corteza terrestre, y otras 2.000 a 3.000 toneladas son liberadas por actividad humana (Foulke 1994 citado por [Moreno Requena 2008)); entre las actividades que mayores emisiones de mercurio genera se encuentra la minería del oro. En el Huila se maneja la minería de oro de filón y de aluvión esta ultima en la cuenca del rio Magdalena; algunas de estas actividades se realizan de manera ilegal en el Departamento (Caracol Radio, 2015), en donde este metal es adicionado a las rocas con el objetivo de formar una amalgama de mercurio oro, para posteriormente eliminar el mercurio y obtener el oro, lo cual conlleva a graves problemas de contaminación de los recursos hídricos en las zonas aledañas en donde se lleva a cabo esta actividad (Unidad de Planeación Minero Energética UPME, 2007).

Básicamente se han establecido tres formas de contaminación con mercurio: Inhalación de los vapores de mercurio metálico, consumo de aguas contaminadas con el metal y consumo de alimentos que estén contaminados con mercurio orgánico (metilmercurio) (Moreno Requena 2008). Hasta cierto punto, todas las formas de mercurio pueden llegar a acumularse; pero el metilmercurio se absorbe y acumula más fácilmente (Olivero \& Jhonson 2002). La biomagnificación del mercurio orgánico es lo que más incide en los efectos para animales y seres humanos. Ha sido estimado que la concentración promedio de metilmercurio es del $90 \%$ del Mercurio total (Hg-T) en los peces de agua dulce según Marrugo-Negrete et al., 2008 citado por (Castro Sanguinetti, 2011).

Los problemas de salud causados por la intoxicación aguda con mercurio metálico y sus compuestos orgánicos se producen por la inhalación de vapores o ingestión de compuestos mercúricos. Por inhalación, se producen lesiones en la mucosa respiratoria, bronquitis y neumonitis química. Por ingestión produce síndrome gastroentérico agudo, insuficiencia renal con anuria y uremia, colitis ulcero hemorrágica, vomito en ocasiones hemorrágico, irritación de la mucosa orofaríngea, posible irritación del esófago. Pasados dos o tres días se produce estomatitis mercurial, la cual se produce porque el mercurio en la saliva irrita e inflama las encías; el ácido sulfhídrico de la fermentación bacteriana produce sulfuro de mercurio negro el cual se revela como ribete gingival. Cuando ocurre la muerte, esta se presenta después de dos o tres días después de la intoxicación por mercurio. Según Sepúlveda et al., 1997, citado por (Machado, et al. 2010). Las intoxicaciones crónicas por mercurio, presenta efectos significativos en la reproducción, y el metilmercurio representa un riesgo especial para los fetos en desarrollo, pues penetra con facilidad la barrera placentaria y puede dañar el sistema nervioso en desarrollo (Programa de las naciones unidas para el medio ambiente, 2002).

Respecto a la normatividad ambiental en Colombia, el Decreto 1594 de 1984 fija los criterios de calidad admisibles para la destinación del recurso para consumo humano, doméstico, recreativo y para la preservación de flora y fauna en aguas dulces; donde el mercurio es considerado una sustancia de interés sanitario, mencionando valores para este, los cuales varían entre $0,02 \mathrm{mg} / \mathrm{L}$ para el agua potable y $0,01 \mathrm{mg} / \mathrm{L}$ para la preservación de flora y fauna; sin embargo no se referencian valores para metilmercurio en agua. En cuanto a la contaminación por mercurio en peces, el Instituto Colombiano de Normas Técnicas y Certificación ICONTEC a través de la Norma 1443 regula las características para consumo humano de pescado fresco, refrigerado, congelado y supercongelado, y establece como máximo valor permisible $0,5 \mu \mathrm{g} / \mathrm{g}$ de mercurio (Mancera Rodríguez \& Álvarez León, 2006).

En Colombia se han llevado a cabo diversos estudios sobre los niveles de mercurio en peces principalmente en las riveras del Rio Magdalena, así como los niveles de este metal en el cabello de las personas que habitan ciertas poblaciones (Ministerio de Ambiente y Desarrollo Sostenible, 2012); sin embargo en el país no se tiene restricción sobre la ingesta diaria tolerable de mer- 
curio total o de metil mercurio en peces; a nivel internacional la EPA(Environmental Protection Agency) define la DRF (Reference Dose o nivel de exposición que no causa efectos adversos en la salud) como 0,1 $\mathrm{\mu g}$ / Kg de peso corporal*día; con el fin de proteger a los fetos humanos de desarrollar efectos perjudiciales y la FDA (The Food and Drug Administration) que define la ADI (Acceptable Daily Intake o nivel de ingestión diaria aceptable) como $0,47 \mu \mathrm{g} / \mathrm{Kg}$ de peso corporal*dia, con el fin de proteger al adulto promedio que es capaz de tolerar los niveles más altos de metilmercurio (Ramos, Estévez, \& Giraldo., 2000); por otro lado la ingesta para mujeres en estado de embarazo y niños de acuerdo al Comité OMS / FAO de expertos conjunta sobre Aditivos Alimentarios (JECFA) recomienda una ingesta semanal tolerable provisional (ISTP) para el metilmercurio de $1.6 \mathrm{mg} /$ $\mathrm{kg}$ de peso corporal / semana (equivalente a 0,20 $\mu \mathrm{g}$ metilmercurio / kg de peso corporal / día) (Minister of Health, 2007).

En el departamento del Huila es escaso el conocimiento que se tiene de la problemática generada por el uso y disposición del mercurio. No hay estudios que indiquen el nivel de esta sustancia en los peces, ni el riesgo al cual puede estar expuesta la población al consumirlo.

Dada la importancia que tiene la especie Pimelodus grosskopfii (capaz) en la idiosincrasia opita, con el plato viudo de capaz y por ende el interés que tiene a nivel comercial en todo el departamento; este proyecto se realizo con el fin de obtener mayor conocimiento sobre los niveles de mercurio y su presencia en esta especie; además de determinar el riesgo al que está expuesta la población huilense al consumirlo y generar una línea base de mercurio para estudios posteriores en la región.

\section{Materiales y métodos}

Elárea de estudio comprende los municipios de Yaguará, Neiva, Hobo y la vereda Puerto Seco en Gigante; lugares donde se pesca capaz (Figura 1); en el municipio de Yaguará las capturas se realizan en la entrada de la Represa de Betania por el sub-sector Brazo Yaguará en la confluencia entre el Rio Yaguará y el Embalse; y en los demás municipios las capturas se realizan sobre el Rio Magdalena, principal arteria fluvial que atraviesa el departamento (IDEAM, 2007). Los principales usos de esta corriente hidrográfica los constituyen el consumo de agua por parte de grupos humanos y ganado; además de irrigación de cultivos, hidroelectricidad, explotación pesquera (Sánchez M. et al., 2001). Y minería aurífera en sus riveras (Diario del Huila, 2013).

Para determinar la concentración de mercurio total en la especie Capaz, se tomaron en total 35 muestras, las cuales fueron proporcionadas por pescadores locales, durante finales del mes de noviembre y principios del mes de diciembre del año 2012; 10 muestras fueron tomadas en la vereda Puerto Seco del municipio de Gigante, 10 en el municipio de Hobo, 8 en el municipio de Neiva y 7 en el municipio de Yaguara.

Los peces fueron pesados, medidos y posteriormente se procedió a realizar un corte tipo filete (Olivero \& Johnson, 2002) estos fueron empacados en doble bolsa Ziplock y congelados (Suchanek, et al., 2008); para ser enviados a la ciudad de Bogotá para su análisis en el laboratorio de Suelos de la Universidad Jorge Tadeo Lozano. La determinación de mercurio se realizó por espectrometría de absorción atómica utilizando generador de hidruros en frío, según la Metodología: AOAC Official Method 977.15 - Mercury in fish.

Para obtener el riesgo al cual está expuesta la población de estudio (pescadores), se determinó a través del índice de riesgo; donde inicialmente se realizó un análisis de la cantidad de consumo de Capaz que se ingiere en estas áreas. Para esto se recurrió a la formulación de encuestas para obtener información general sobre la población de estudio, con el fin de identificar las tendencias del consumo de capaz.

La cantidad de encuestas realizadas a los pescadores se definió según la ecuación del tamaño de muestra con un intervalo de confianza del 95\%; entrevistando 38 pescadores en Yaguará, 10 en gigante, 23 en Hobo y 8 en Neiva.

Para determinar el nivel de ingesta de metil mercurio por kilogramo de peso corporal se determinó de acuerdo a la fórmula propuesta por el Programa de las Naciones Unidas para el medio ambiente, 2010 ver ecuación 1 ; tomando como referencia de peso corporal en $70 \mathrm{Kg}$; y la cantidad de metil mercurio como el 90\% del mercurio total encontrado en peces según Marrugo- Negrete

Ecuación 1. Ingesta de metil mercurio

Ingesta de $\mathrm{MeH}=\quad$ [Cantidad de pez consumido(semana)*(Concentración MeHg( $\mu$ g/Kg)]

[(Kilogramo de peso corporal $(\mathrm{Kg})]$ 


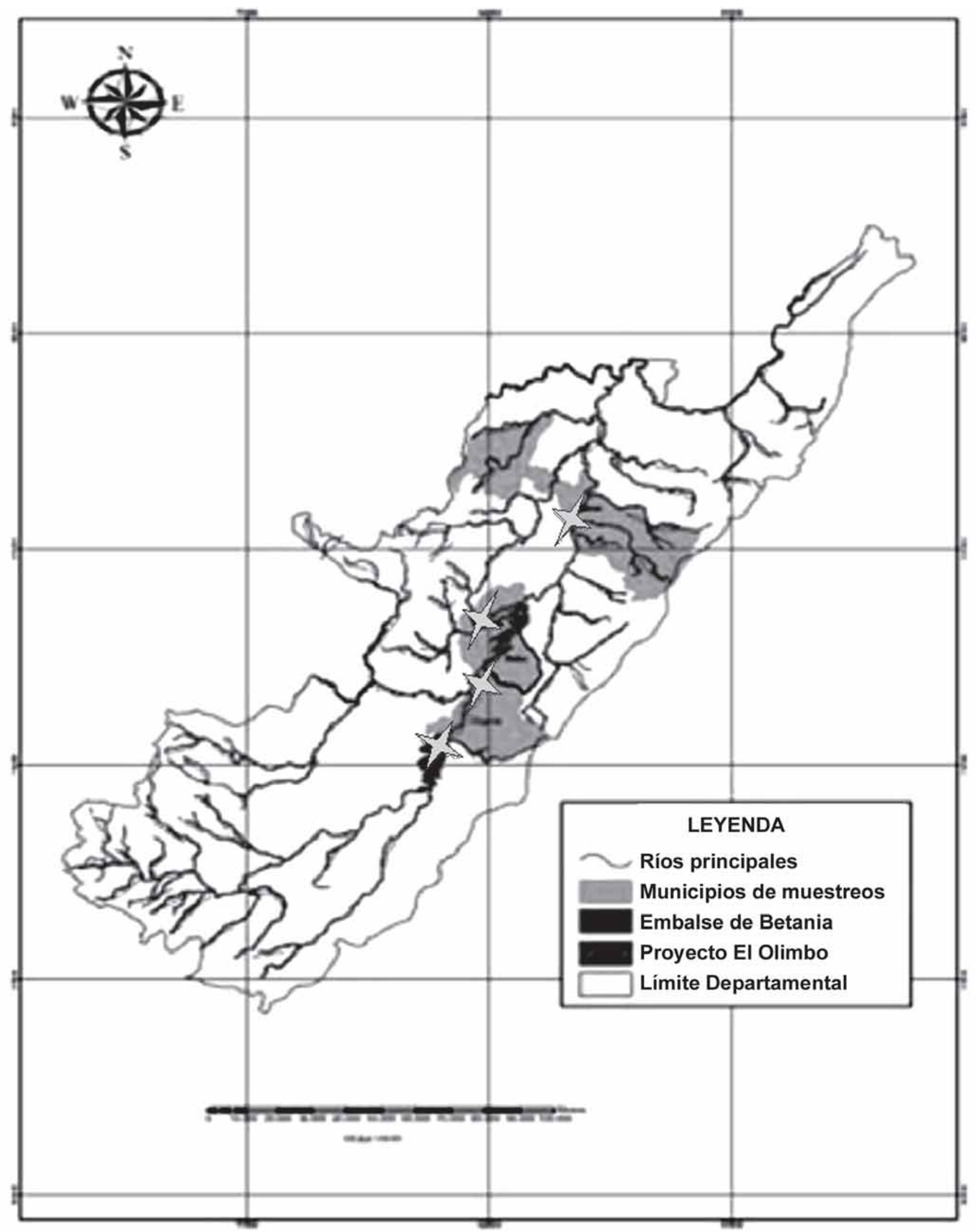

Figura1. Puntos de muestreo. Fuente: Corporación Autónoma Regional del Alto Magdalena CAM. 
et al., 2008 citado por (Castro Sanguinetti, Facultad de Medicina Veterinaria-UNMSM Perú, 2011).

Con la ingesta obtenida por municipio se procedió a desarrollar la obtención del índice de riesgo "Hazard Quotient” (HQ) al que se encuentra expuesta la población de estudio (Trujillo, et al., 2012).

\section{Resultados y discusión}

De las 35 muestras tomadas de la especie, todas reportaron niveles de mercurio; a los datos obtenidos se les realizo un análisis estadístico que consistió en una prueba de Kruskal Wallis, que equivale a un ANOVA de una sola vía no paramétrico, debido a que se tienen 10 o menos datos por muestras y a que los datos tuvieron que ser normalizados antes del análisis, obteniendo como resultado que no hay una variación significativa entre las muestras con un valor de significancia del 0.05 , esto indica que estadísticamente los cuatros puntos presentan concentraciones de mercurio similares (Figura 2).
El lugar que reporto el mayor nivel de mercurio total en la especie de estudio, fue el municipio de Hobo el cual tuvo un promedio de $78.54 \mathrm{Hg}-\mathrm{T}$ ( $\mu \mathrm{g} \mathrm{Hg} / \mathrm{kg}$ de muestra peso húmedo), seguido se encuentra la vereda Puerto Seco en el municipio de Gigante con un promedio de $76.10 \mathrm{Hg}-\mathrm{T}$ ( $\mu \mathrm{g} \mathrm{Hg} / \mathrm{kg}$ de muestra peso húmedo), Neiva con 65.04 Hg-T ( $\mu \mathrm{g} \mathrm{Hg} / \mathrm{kg}$ de muestra peso húmedo) y el lugar que reporto la menor cantidad de mercurio fue Yaguará con un promedio de $36.2 \mathrm{Hg}$ $\mathrm{T}$ ( $\mu \mathrm{g} \mathrm{Hg} / \mathrm{kg}$ de muestra peso húmedo); de acuerdo con Hakansson 1984 citado por (Olivero \& Jhonson, 2002) concentraciones superiores a $75 \mu \mathrm{gHg} / \mathrm{Kg}$ en especies acuáticas pueden ser asignadas a contaminación por actividades de tipo antropogénico, resultados que se pueden atribuir a la presencia de actividad minera para la obtención de oro y/o al uso excesivo del mercurio durante el proceso de beneficio para la adquisición de este, debido a que no se presentan otras posibles fuentes de emisión de este metal; ya que las emisiones producto de la formulación de agroquímicos son descartadas, porque en la zona de estudio se cultiva principalmente café, cacao, caña panelera, frutales, y pastos dedicados a la ganadería, cultivos de los cuales

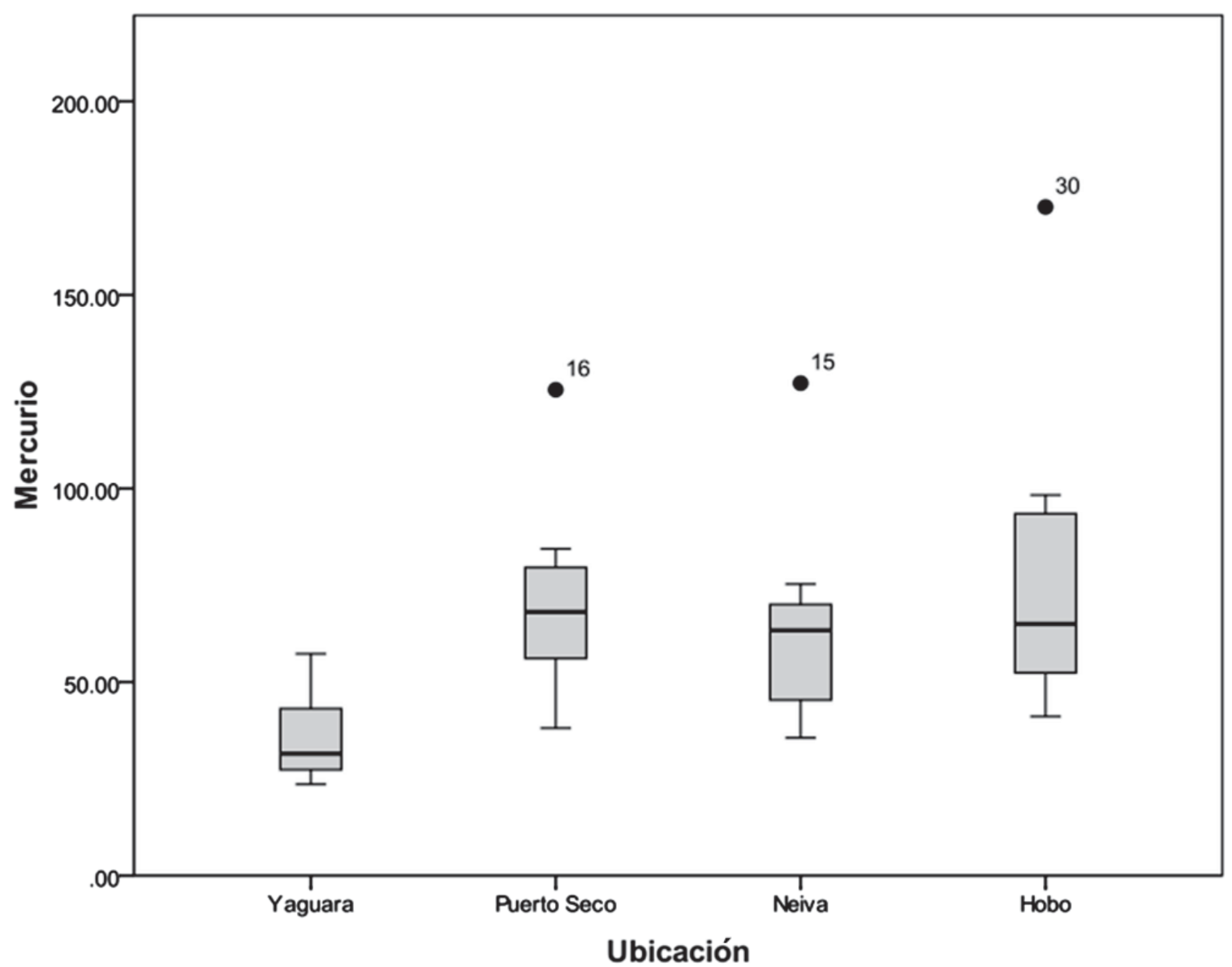

Figura 2. Concentración de mercurio total. Fuente: Autores del documento. 
no se tienen registros que utilicen mercurio en sus agroquímicos, este metal se encuentra presente en algunos agroquímicos empleados para la papa y cereales (Programa de las Naciones Unidas para el medio ambiente PNUMA, 2002).

En cuanto a la evaluación del riesgo en la población se desarrolló primero la frecuencia de consumo en las diferentes poblaciones, además de la cantidad de pescado ingerido por semana (Kg* Semana) y con estos datos se obtuvo la ingesta semanal de metilmercurio ( $\mu \mathrm{g} \mathrm{MeHg} / \mathrm{Kg} *$ Semana).
Para determinar la ingesta por población se realizó un promedio entre los resultados de ingesta en cada muestra por zona ( Tabla 1, 2, 3, y 4).

Existe en la bibliografía internacional algunos niveles máximos permisibles o recomendados de metilmercurio en el pescado, según el JECFA (Comité mixto OMS/FAO de expertos en aditivos alimentarios), citado por (Programa de las naciones unidas para el medio ambiente, 2002) sostiene como ingesta semanal tolerable provisional 3,3 $\mu \mathrm{g}$ de $\mathrm{MeHg} / \mathrm{Kg}$ por kilo de peso por semana.

Tabla 1. Ingesta de metil-mercurio Yaguará.

\begin{tabular}{ccccccc}
\hline Peso (gr.) & $\begin{array}{c}\text { Cantidad Hg- } \\
\boldsymbol{\mu g ~ H g} / \mathbf{K g} \\
\text { de muestra }\end{array}$ & $\begin{array}{c}\text { Cantidad MeHg- } \\
\boldsymbol{\mu g} \text { Mehg/ Kg } \\
\text { de muestra }\end{array}$ & $\begin{array}{c}\text { Frecuencia } \\
\text { de consumo } \\
\text { (Semanas) }\end{array}$ & $\begin{array}{c}\text { Cantidad } \\
\text { ingerido } \\
\text { (Kg* Semana) }\end{array}$ & $\begin{array}{c}\text { Ingesta de MeHg } \\
\text { Semanal } \mathbf{\mu g M e H g} / \\
\text { Kg.sem }\end{array}$ & $\begin{array}{c}\text { Índice de riesgo } \\
\text { Hazard Quotient } \\
\text { (HQ) }\end{array}$ \\
\hline 23 & 25,1 & 22,59 & & 0,02 & 0,01 & 0,01 \\
67,4 & 57,3 & 51,57 & & 0,05 & 0,04 & 0,05 \\
35,7 & 31,5 & 28,35 & & 0,03 & 0,01 & 0,01 \\
65,1 & 49,1 & 44,19 & 0,71 & 0,05 & 0,03 & 0,04 \\
73,8 & 37,2 & 33,48 & & 0,05 & 0,03 & 0,04 \\
38,5 & 29,6 & 26,64 & & 0,03 & 0,01 & 0,01 \\
43,5 & 23,6 & 21,24 & & 0,03 & 0,01 & 0,01 \\
\hline
\end{tabular}

Fuente: Autores del documento.

Tabla 2. Ingesta de metil mercurio en Gigante-Vereda Puerto Seco.

\begin{tabular}{ccccccc}
\hline Peso (gr.) & $\begin{array}{c}\text { Cantidad Hg- } \\
\boldsymbol{\mu g ~ H g} / \mathbf{K g} \\
\text { de muestra }\end{array}$ & $\begin{array}{c}\text { Cantidad MeHg- } \\
\boldsymbol{\mu g} \text { Mehg / Kg } \\
\text { de muestra }\end{array}$ & $\begin{array}{c}\text { Frecuencia } \\
\text { de consumo } \\
\text { (Semanas) }\end{array}$ & $\begin{array}{c}\text { Cantidad } \\
\text { ingerido } \\
\text { (Kg* Semana) }\end{array}$ & $\begin{array}{c}\text { Ingesta de MeHg } \\
\text { Semanal } \mathbf{\mu g M e H g} \text { / } / \text { Índice de riesgo } \\
\text { Kg.sem }\end{array}$ & $\begin{array}{c}\text { Hazard Quotient } \\
\text { (HQ) }\end{array}$ \\
\hline 72,50 & 125,50 & 112,95 & & 0,20 & 0,33 & 0,47 \\
96,80 & 84,40 & 75,96 & & 0,27 & 0,29 & 0,42 \\
44,90 & 38,10 & 34,29 & & 0,13 & 0,06 & 0,09 \\
47,80 & 123,10 & 110,79 & & 0,13 & 0,21 & 0,30 \\
103,70 & 79,60 & 71,64 & & 0,29 & 0,30 & 0,42 \\
255,60 & 77,80 & 70,02 & 2,80 & 0,72 & 0,72 & 1,02 \\
80,00 & 68,10 & 61,29 & & 0,22 & 0,20 & 0,28 \\
136,50 & 63,20 & 56,88 & & 0,38 & 0,31 & 0,44 \\
78,90 & 45,10 & 40,59 & & 0,22 & 0,13 & 0,18 \\
88,80 & 56,10 & 50,49 & & 0,25 & 0,18 & 0,26 \\
\hline
\end{tabular}

Fuente: Autores del documento. 
Tabla 3. Ingesta de metil mercurio Neiva.

\begin{tabular}{ccccccc}
\hline Peso (gr.) & $\begin{array}{c}\text { Cantidad Hg- } \\
\boldsymbol{\mu g ~ H g} / \mathbf{K g} \\
\text { de muestra }\end{array}$ & $\begin{array}{c}\text { Cantidad MeHg- } \\
\boldsymbol{\mu g} \text { Mehg / Kg } \\
\text { de muestra }\end{array}$ & $\begin{array}{c}\text { Frecuencia } \\
\text { de consumo } \\
\text { (Semanas) }\end{array}$ & $\begin{array}{c}\text { Cantidad } \\
\text { ingerido } \\
\text { (Kg* Semana) }\end{array}$ & $\begin{array}{c}\text { Ingesta de MeHg } \\
\text { Semanal } \mathbf{~ M g M H g} / \\
\text { Kg.sem }\end{array}$ & $\begin{array}{c}\text { Índice de riesgo } \\
\text { Hazard Quotient } \\
\text { (HQ) }\end{array}$ \\
\hline 210,00 & 75,30 & 67,77 & & 0,45 & 0,43 & 0,62 \\
137,10 & 46,60 & 41,94 & & 0,29 & 0,17 & 0,25 \\
180,80 & 64,50 & 58,05 & & 0,39 & 0,32 & 0,46 \\
168,30 & 35,60 & 32,04 & & 0,36 & 0,16 & 0,23 \\
61,90 & 64,80 & 58,32 & 2,13 & 0,13 & 0,11 & 0,16 \\
122,30 & 62,20 & 55,98 & & 0,26 & 0,21 & 0,30 \\
50,50 & 44,10 & 39,69 & & 0,11 & 0,06 & 0,09 \\
105,93 & 127,20 & 114,48 & & 0,23 & 0,37 & 0,53 \\
\hline
\end{tabular}

Fuente: Autores del documento.

Tabla 4. Ingesta de metil mercurio Hobo.

\begin{tabular}{ccccccc}
\hline Peso (gr.) & $\begin{array}{c}\text { Cantidad Hg- } \\
\boldsymbol{\mu g ~ H g} / \mathbf{~ K g} \\
\text { de muestra }\end{array}$ & $\begin{array}{c}\text { Cantidad MeHg- } \\
\boldsymbol{\mu g} \text { Mehg / Kg } \\
\text { de muestra }\end{array}$ & $\begin{array}{c}\text { Frecuencia } \\
\text { de consumo } \\
\text { (Semanas) }\end{array}$ & $\begin{array}{c}\text { Cantidad } \\
\text { ingerido } \\
\text { (Kg* Semana) }\end{array}$ & $\begin{array}{c}\text { Ingesta de MeHg } \\
\text { Semanal } \mathbf{\mu g M e H g} \text { / } \\
\text { Kg.sem }\end{array}$ & $\begin{array}{c}\text { Índice de riesgo } \\
\text { Hazard Quotient } \\
\text { (HQ) }\end{array}$ \\
\hline 66,1 & 54,9 & 49,41 & & 0,12 & 0,09 & 0,12 \\
77 & 72,3 & 65,07 & & 0,14 & 0,13 & 0,19 \\
82,4 & 98,3 & 88,47 & & 0,15 & 0,19 & 0,28 \\
58,5 & 52,4 & 47,16 & & 0,11 & 0,07 & 0,1 \\
84,7 & 172,7 & 155,43 & 1,85 & 0,16 & 0,35 & 0,5 \\
79,7 & 57,7 & 51,93 & & 0,15 & 0,11 & 0,16 \\
63,9 & 41,1 & 36,99 & & 0,12 & 0,06 & 0,09 \\
59,9 & 50 & 45 & & 0,11 & 0,07 & 0,1 \\
41 & 93,5 & 84,15 & & 0,08 & 0,09 & 0,13 \\
42,6 & 92,5 & 83,25 & & 0,08 & 0,09 & 0,13 \\
\hline
\end{tabular}

Fuente: Autores del documento.

De igual manera Hirsch, 2002 y Ordiano Flores, 2009 establecen que La FDA (Food and drug administration- administración de alimentos y fármacos) y la agencia de protección ambiental de EEUU adoptan un límite más estricto de consumo tolerable de MeHg (0.4 y $0.1 \mu \mathrm{g}$ día- $1 \mathrm{~kg}-1$ de peso corporal, respectivamente, para toda la población) (Escobar Sánchez, 2010).

El método de cálculo del índice de riesgo (HQ) fue estimado de la relación entre la ingesta semanal promedio de metilmercurio MeHg en cada población y la dosis de referencia establecida por IRIS, 1995; (Trujillo, et al.), el valor DRF para el MeHg es de 0,0001 mg/Kg*día. Para este trabajo se convirtió la dosis de referencia a $\mu \mathrm{g} / \mathrm{Kg}$ *Semana. Estos resultados se muestran en la siguiente tabla 5 .

Se estima que un HQ mayor a 1.0 indica potenciales efectos negativos para la salud; es claro que este índice varía según las concentraciones de ingesta, de productos que contengan mercurio, para este caso la frecuencia de consumo de Capaz (Marrugo, Lans, \& Benítez, 2007) y (Trujillo, et al.). 
Tabla 5. Índice promedio de riesgo.

\begin{tabular}{lccc}
\hline $\begin{array}{l}\text { Municipio } \\
\text { de estudio }\end{array}$ & $\begin{array}{c}\text { RfD para MeHg } \\
\mathbf{\mu g} / \mathbf{K g}^{*} \text { Sem }\end{array}$ & $\begin{array}{c}\text { Ingesta de MeHg semanal } \\
\mathbf{\mu g ~ M e H g} / \text { Kg.sem }\end{array}$ & $\begin{array}{c}\text { Índice de riesgo } \\
\text { HQ = E / DRF }\end{array}$ \\
\hline Yaguara & 0,7 & 0,03 & 0,04 \\
Gigante & & 0,39 & 0,56 \\
Neiva & 0,33 & 0,47 \\
Hobo & 0,18 & 0,26 \\
\hline
\end{tabular}

Se puede observar que los cuatro municipios se encuentran en un riesgo aceptable, sin posibles efectos para la salud; sin embargo no podemos descarta que presenten algún tipo de riesgo en la población ya que si el consumo de pez supera de manera elevada el nivel de ingesta tolerable podrían alcanzar y superar el índice de riesgo, con lo cual se incrementa el riego de padecer alteraciones y efectos devastadores en la salud. Al ser el consumo de pescado el medio más importante de exposición es necesario hacer un control a la dieta de la población para evitar que el efecto del metilmercurio sea severo e irreparable.

El Municipio con menor índice de riesgo es el municipio de Yaguará, el cual reporto igualmente en la determinación de mercurio total Hg-T, un valor inferior al resto de los municipios en donde se realizaron los muestreos. Con esto se puede deducir la relación existente entre la concentración de mercurio total Hg-T y el índice de riesgo HQ, es decir, a menor concentración de mercurio se va a obtener una menor cantidad de ingesta del metal lo cual va a generar que el índice de riesgo sea menor en población.

\section{Conclusiones}

Del total de muestras recolectadas se logro establecer la existencia de mercurio en los peces Pimelodus grosskopfii, en el departamento del Huila.

En los cuatros puntos en que se realizó el índice de riesgo se obtuvo un HQ inferior a uno, lo cual indica un riesgo aceptables de exposición; aunque se debe cuidar la cantidad de ingesta semanal con el fin de no afectar la salud de la población Huilense.

Aunque existen niveles bajos de mercurio en las zonas de estudio, las poblaciones o persona expuestas a ni- veles bajos de mercurio inorgánico pueden desarrollar alteraciones en las funciones del sistema nervioso con consecuencias neuro-fisiológicas graves en especial en los fetos y niños.

No se estableció un relación directa entre la presencia de mercurio y la minería del oro; es evidente que la extracción de este metal, esta afectando los recursos hídricos, y es necesario tomar medidas tendientes a detener la contaminación por mercurio de este recurso natural.

\section{Referencias bibliográficas}

1. Alcaldía de Gigante, (22 de 05 de 2013). Pagina de la alcaldía de Gigante. Recuperado el 1 de 06 de 2013, de Documentos sobre el municipio: http:// www.gigante-huila.gov.co/nuestromunicipio. shtml?apc $=\mathrm{mIxx}-1-\& \mathrm{~m}=\mathrm{d}$

2. Alcaldía de Hobo. (25 de 04 de 2013). Alcaldía de Hobo. Recuperado el 26 de 04 de 2013, de Alcaldía de Hobo: http://www.alcaldiahobo.gov.co

3. Alcaldía de Yaguara. (2013). Alcaldía de Yaguara. Recuperado el 24 de 05 de 2013, de http://yaguarahuila.gov.co

4. Banco de la Republica, C. (2011). Sitio web Banco de la Republica, Colombia. Recuperado el 17 de 12 de 2011, de Series estadísticas de metales preciosos: http://www.banrep.gov.co/series-estadisticas/ see_met_prec_dia.htm

5. Barceló. (2000). TESIS DOCTORADO: ESTUDIO DE LA MOVILIDAD DE Ca, Cd, Cu, Fe, Mn, Pb y Zn en sedimentos de la presa José Antonio Alzae en el Estado de México. México: Universidad Autónoma del Estado de México. 
6. Buitrago, C. (1976). Ocurrencias Minerales del Departamento del Huila. INGEOMINAS. Ibagué: INGEOMINAS.

7. Caracol Radio. (19 de Marzo de 2015). Golpe a minería ilegal se registra en el Huila. Caracol Radio Regionales.

8. Castro Sanguinetti, G. (2011). Facultad de Medicina Veterinaria-UNMSM Perú. Retrieved 2013 06Junio Fromm http://veterinaria.unmsm.edu.pe/files/ Articulo_castro_efecto_mercurio.pdf

9. Castro Sanguinetti, G. (2011). http://veterinaria. unmsm.edu.pe/. Fromm http://veterinaria.unmsm. edu.pe/files/Articulo_castro_efecto_mercurio.pdf

10. Centro provincial de Gestión Minero Agroempresarial CPGMAE Alto Nordeste Antioqueño. (2007). Diagnostico ambiental para la explotación, explotación y beneficio de oro en el sector minero de Pacarni, entre Tesalia e Iquira departamento del Huila. Centro provincial de Gestión Minero Agroempresarial CPGMAE- Alto Nordeste Antioqueño. Neiva: Gobernación del Huila.

11. Diario del Huila. (23 de 05 de 2013). Pagina Web Diario del Huila. Recuperado el 21 de 07 de 2013, de http://www.diariodelhuila.com/site/index.php? option=com_content\&view=article\&id=35947: incautan-maquinaria-para-extraccion-de-oro\&catid= 25:judicial\&Itemid $=15$

12.Escobar Sánchez, O. (Diciembre de 2010). Bioacumulación y biomagnificación de mercurio y selenio en peces pelágicos mayores de la costa occidental de baja California sur, México . México, México.

13.Gammons, C., Slotton, D., Gerbrandt, B., Weight, W., Young, C., McNearny, R., y otros. (2006). Mercury concentrations of fish, river water, and sediment in the Rio Ramis-Lake Titicaca watershed, Perú . Science of the Total Environment (368), 637-648.

14. Gobernación del Huila. (2003). Anuario Estadístico del Huila. Gobernación del Huila, Huila. Neiva: Gobernación del Huila.

15.Griem, W., \& Griem-Klee, S. (16 de 06 de 2011). Universidad de Atacama. Recuperado el 18 de 10 de 2011, de Apuntes Geología General: http://www. geovirtual.cl/EXPLORAC/TEXT/03000g 1.htm\# M\%E9todo\%20geoqu\%EDmico\%20de\%20 exploraci\%F3n
16.Güiza, L., \& Aristizábal, J. D. (2013). Scielo Colombia - Scientific Electronic Library Online. Retrieved 2013 27-Mayo Fromm http://www.scielo. org.co/scielo.php?pid=S0122-74832013000100002 \&script=sci_arttext

17.Higueras, R., \& Oyarzun, P. (1984). Prospección geoquímica: conceptos básicos Yacimientos Minerales,. Centro de Estudios Ramón Areces,. Madrid: R. Lunar \& R. Oyarzun.

18.IDEAM, C. Y. (2007). Nueva medición de la calidad de agua.

19.Instituto de Hidrología, Meteorología y Estudios Ambientales. (2010). Leyenda Nacional de Coberturas de la Tierra Metodología CORINE Land COVER adaptada para Colombia Escala 1:100.000. Bogotá: IDEAM.

20.J. L., A, O., R, F.-M., \& Rucandio. (2008). Biodisponibilidad del mercurio en suelos contaminados por explotaciones mineras. Recuperado el 18 de 10 de 2011, de http://ingenierosdeminasdelevante. org/documentos/biodisponibilidad_mercurio.pdf

21.Loredo, L., Ordoñez, A., Fernandez-Martinez, \& Rucandio. (2008). Biodisponibilidad del mercurio en suelos contaminados por explotaciones mineras. Recuperado el 18 de 10 de 2011, de http: //ingenierosdeminasdelevante.org/documentos/ biodisponibilidad_mercurio.pdf

22. Machado, L. G., Ospina, J. H., Henao, N. A., \& Marin, F. D. (Enero de 2010). Problemática ambiental ocasionada por el mercurio proveniente de la minería aurífera tradicional, en el corregimiento de Providencia, Antioquia. Medellín.

23. Machado, L., Ospina, J., Henao, N., \& Marin, F. (2010). Tesis: Problemática ambiental ocasionada por el Mercurio proveniente de la minería aurífera tradicional, en el corregimiento de Providencia, Antioquia. Medellín. Medellín, Antioquia, Colombia: Universidad de Antioquia.

24.Mancera Rodríguez, N. J., \& Álvarez León, R. (2006). Estado del conocimiento de las concentraciones de mercurio y otros metales pesados en peces dulceacuícolas de Colombia. Acta Biológica Colombiana , 11 (1), 3-23.

25.Mancera Rodríguez, N. J., \& Cala, P. (1997). Asociación Colombiana de Ictiólogos. Retrieved 2013 
30-Junio from http://www.acictios.org/Dahlia\%202/ Revista\%202,\%20Art.2\%20Macera\%20y\% 20Cala.\%20ASPECTOS\%20BIOECOLOGICOS\% 20DE\%20LA\%20COMUNIDAD.pdf

26.Marrugo, J., Lans, E., \& Benítez, L. (15 de Junio de 2007). Hallazgo de mercurio en peces de la ciénaga de Ayapel, Córdoba, Colombia.

27.Minister of Health. (2007). Human Health Risk Assessment of Mercury in Fish and Health Benefits of Fish Consumption. Ontario: Health Canadá .

28.Ministerio de Ambiente y Desarrollo Sostenible. (2012). Diagnostico Nacional de Salud Ambiental. Bogotá: Ministerio de Ambiente.

29.Ministerio del Medio Ambiente. (2002 Febrero). CORTOLIMA-Corporación Autónoma Regional del Tolima. Retrieved 2013 14-Junio from http://www. cortolima.gov.co/SIGAM/cartillas/Gestion\% 20minero \%20ambiental.pdf

30. Montenegro, G. (2005). Pagina Universidad Escuela Superior Politécnica del Litoral. Recuperado el 2011, de CIB Centro de Información bibliotecario: http://www.cib.espol.edu.ec/Digipath/Librospdf/ Libro\%20Geoqu\%C3\%ADmica.pdf

31. Moreno Requena, J. A. (2008). Alteraciones comportamentales y de personalidad debido a la exposición ocupacional a mercurio en un grupo de mineros del oro de la región del Bagre- Antioquia. Medellín.

32.Muñoz, F., \& Delgado, J. (2006). Tesis: Determinación de mercurio en suelos de Bucaramanga, utilizando un pirolizador acoplado a un detector de mercurio basado en espectroscopía de absorción atómica diferencial de ZEEMAN. Bucaramanga: Universidad Industrial de Santander.

33. Neiva, A. d. (02 de Junio de 2013). Alcaldía de Neiva. Recuperado el 20 de Junio de 2013, de http:// www.alcaldianeiva.gov.co

34. Olivero, J., \& Jhonson, B. (2002). El lado gris de la minería del oro: La contaminación con Mercurio en el norte de Colombia. Cartagena: Universidad de Cartagena.

35.OMS. (2012 Abril). OMS - El mercurio y la salud. Retrieved 2013 28-Febrero from http://www.who. int/mediacentre/factsheets/fs361/es/
36.Programa de las naciones unidas para el medio ambiente. (19 de Octubre de 2010). Resumen ejecutivo del documento sobre orientación para identificar poblaciones en situación de riesgo de exposición a mercurio.

37. Programa de las naciones unidas para el medio ambiente. (Diciembre de 2002). Evaluación mundial sobre el mercurio . Ginebra, Suiza.

38. Ramos, C. X., Estévez, S. L., \& Giraldo., E. (2000). Nivel de contaminación por metilmercurio en la región de la Mojana. Nivel de contaminación por metilmercurio en la región de la Mojana . Bogotá, Colombia.

39. Repetto, M. (2009). Toxicología Fundamental. Sevilla: Ediciones Díaz de Santos.

40.Sánchez Alarcón, S. R. (2005). Universidad Mayor de San Andrés Bolivia . Retrieved 2013 15-Julio from http://bibliotecadigital.umsa.bo:8080/rddu/ bitstream/123456789/408/1/TM578.pdf

41.Sánchez Ramírez, M. Biodiversidad en ecosistemas acuáticos del Alto Magdalena en el Huila. En A. Olaya Amaya, \& M. Sánchez Ramírez, Ecosistemas Estratégicos del Huila.

42. Standard Methods, f. W. (1999). Standard Methods for Water and Wastewater Examination - 20th. Washington D.C: American Public Health Association.

43. Trujillo, F., Lasso, C. A., Diazgranados, M. C., Farina, O., Pérez, L. E., Barbarino, A. (29 de 10 de 2012). Fundación Omacha. Recuperado el 14 de 07 de 2013, de www.omacha.org: http://www.omacha.org/publicaciones/art-cientificos

44. Trujillo, F., Lasso, C. A., Diezgranados, M. C., Farina, O., Pérez, L. E., Barbarino, A. (s.f.). Evaluación de la contaminación por mercurio en peces de interés comercial y de la concentración de organoclorados y organofosforados en el agua y sedimentos de la Orinoquia . Colombia .

45.Unidad de Planeación Minero Energética UPME. (2007). Producción mas limpia en la minería del oro en Colombia Mercurio, Cianuro y otras sustancias. Bogotá, Colombia: Unidad de Planeación Minero Energética.

45.Valbuena, R. D., Vásquez, W., Zapata, B., \& Gutiérrez, M. C. (2010). Aspectos preliminares sobre nutrición y alimentación en Capaz (pimelodus grosskopfii). Neiva: Gobernación del Huila y Universidad Surcolombiana. 